CAUSATION

\title{
Frequent nut consumption reduced the risk of coronary heart disease in women
}

\author{
Hu FB, Stampfer MJ, Manson JE, et al. Frequent nut consumption and risk of coronary heart disease in women: prospective cohort \\ study. BMJ 1998 Nov 14;317:1341-5.
}

\section{Question}

Is nut consumption associated with coronary heart disease $(\mathrm{CHD})$ in women?

\section{Design}

Cohort study of women in the Nurses' Health Study with follow up of 14 years.

\section{Setting \\ USA.}

\section{Participants}

86016 women aged 34-59 years who were registered nurses. Exclusion criteria were previously diagnosed cancer, angina, myocardial infarction, stroke, or other cardiovascular diseases; or implausibly high or low scores for total energy intake. Follow up was $98 \%$.

\section{Assessment of risk factors}

Frequency of nut consumption and total energy intake (self reported using dietary questionnaires); smoking; age; body mass index; alcohol; use of menopausal hormones, multivitamins, vitamin E, and aspirin; and exercise.

\section{Main outcome measures}

Fatal or non-fatal myocardial infarction, or other fatal CHD verified by electrocardiogram or cardiac enzymes.

\section{Main results}

1255 major CHD events occurred during 14 years of follow up: 861 were cases of non-fatal myocardial infarction and 394 were cases of fatal CHD. Multivariate analysis showed that women who ate $\geqslant 140 \mathrm{~g}(\geqslant 5 \mathrm{oz})$ of nuts each week compared with women who ate $<28 \mathrm{~g}(<1 \mathrm{oz})$ each week had a lower risk for total CHD (relative risk $0.65,95 \% \mathrm{CI} 0.47$ to 0.89 ). These women did not have a lower risk of fatal CHD or non-fatal myocardial infarction. Tests of linear trend across increasing categories of nut consumption showed lower risks for total CHD ( $\mathrm{p}$ for trend $<0.001$ ), fatal CHD ( $\mathrm{p}$ for trend $=0.007$ ), and non-fatal myocardial infarction ( $\mathrm{p}$ for trend $=0.04$ ) as nut consumption increased from $28 \mathrm{~g}(1 \mathrm{oz})$ each week to $\geqslant 140 \mathrm{~g}(\geqslant 5 \mathrm{oz})$ each week. These results remained the same after further adjustment for potential dietary confounding variables (eg, saturated fat, polyunsaturated fat, trans-fat, fibre, vegetables, and fruits) and within subgroups of potential confounding variables.

\section{Conclusion}

Frequent nut consumption was associated with a reduced risk of coronary heart disease in women who were registered nurses.

Source of funding: US National Institute of Health.

For correspondence:Dr F Hu, Department of Nutrition, Harvard School of Public Health, Boston, MA 02115, USA.Fax +1 6174322435 .

\section{Commentary}

This study by $\mathrm{Hu}$ et al examined the relation between increased nut consumption and decreased risk of CHD in women who were nurses in the US. The results of this study suggest that women who frequently consumed nuts had an approximately $35 \%$ lower risk of coronary disease than women who rarely ate nuts. This information is consistent with several other previously published studies..$^{12} \mathrm{Hu}$ et al also addressed the question of whether people who consumed nuts maintained a relatively healthier lifestyle and found that even when taking these factors into account, a definite risk reduction persisted in this population of women who were nurses.

The weaknesses of this study are those inherent in self reported dietary intake: underestimation and imprecision. ${ }^{3}$ An observational study lacks the method- ological rigour of a randomised controlled trial. Also, nurses represent a biased sample because of their education and occupation, which limits the generalisability of the findings.

The exact mechanism by which nuts protect against coronary heart disease is not yet known. The authors suggest various mechanisms including the beneficial effect of unsaturated fats on serum lipids $^{4}$; the role of nitric oxide in inhibiting platelet adhesion; the antithrombotic and antiarrhythmic effects of linolenic acid; and the high content of magnesium, copper, folic acid, protein, potassium, fibre, and vitamin $\mathrm{E}$ in nuts.

These research findings need to be applied in combination with other healthy lifestyle patient education. The inclusion of nuts in a healthy diet should be discussed as an alternative protein.
Moderate consumption of nuts can be incorporated into a healthy and balanced diet, although more research is required to explain the exact mechanism of risk reduction for coronary disease.

Vaska Micevski, RN, MScN Acute Care Nurse Practitioner Cardiac Program Researcher, Women's Health Program The Toronto Hospital Toronto, Ontario, Canada

1 Fraser GE, Shavlik DJ. Risk factors for all-cause and coronary heart disease mortality in the oldest-old. The Adventist health study. Arch Intern Med 1997;157:2249-58

2 Prineas RJ, Kushi LH, Folsom AR, et al. Walnuts and serum lipids. NEngl J Med 1993;329:35960.

3 Schoeller DA. Limitations in the assessment of dietary energy intake by self-report. Metabolism 1995;44(suppl 2):18-22.

4 Dreher ML, Maher CV, Kearney P. The traditional and emerging role of nuts in healthful diets. Nutr Rev 1996:54;241-5. 